\title{
Gravitational Fields and Gravitational Waves tony1807559167@gmail.com
}

\author{
Tony Yuan \\ Beihang University, China
}

January 3, 2022

\begin{abstract}
The relative velocity between objects with finite velocity affects the reaction between them. This effect is known as general Doppler effect. The Laser Interferometer Gravitational-Wave Observatory (LIGO) discovered gravitational waves and found their speed to be equal to the speed of light $c$. Gravitational waves are generated following a disturbance in the gravitational field; they affect the gravitational force on an object. Just as light waves are subject to the Doppler effect, so are gravitational waves. This article explores the following research questions concerning gravitational waves: Is there a linear relationship between gravity and velocity? Can the speed of a gravitational wave represent the speed of the gravitational field (the speed of the action of the gravitational field upon the object)? What is the speed of the gravitational field? What is the spatial distribution of gravitational waves? Do gravitational waves caused by the revolution of the Sun affect planetary precession? Can we modify Newton's gravitational equation through the influence of gravitational waves?
\end{abstract}

Keywords: Newtonian gravity; Doppler effect; gravitational wave; gravitational field; LIGO; gravitational constant; precession of the planets

\section{Introduction}

Newtonian gravity ${ }^{[1][2]}$ is a force that acts at a distance. No matter how fast an object travels, gravity acts upon the object instantaneously. Gravity is only related to the mass and distance of the object, equal to $\frac{G_{0} M m}{r^{2}}$, of which the universal gravitational constant ${ }^{[3]} G_{0}=6.67259 \times 10^{-11} \mathrm{Nm}^{2} / \mathrm{kg}^{2} . G_{0}$ is measured when two objects are relatively stationary. This can be regarded as a static gravitational constant. Newtonian gravity states that the speed of the gravitational field on an object is infinite, therefore, whether two objects are relatively stationary or moving, both can be considered unchanged, so there is no general Doppler effect ${ }^{[4]}$. The Laser Interferometer Gravitational-Wave Observatory (LIGO) ${ }^{[5]}$ first discovered gravitational waves ${ }^{[6][7]}$ and measured their speed. This discovery thus leads us to consider whether the speed of gravitational field ${ }^{[8]}$ is the same as that of the gravitational wave. We know that when we put a stone into the water, in addition to causing slow water waves, it will also cause 
sound waves in the water, the speed will be much greater than that of water waves. So the speed of the water waves we observe cannot represent that of sound waves in the water. Will gravitational waves be like this?

General relativity (GR), it's view on the speed of gravity is different from that of Newton and Laplace. GR also believes that the speed of gravity is equal to the speed of light, but until now, scientists have been unable to prove this view. But we know if the gravitational field has a finite speed, there will be a general Doppler effect between the gravitational field and the object. To determine the speed of the gravitational field, we assume the speed of the gravitational field is equal to the speed of light. For the convenience of analysis, we use $X$ to represent the speed of the gravitational field. If the planetary motions of the solar system calculated under this hypothesis are consistent with astronomical observations, the correctness of this hypothesis can be proved, otherwise it is proved that the speed of the gravitational field is not equal to that of light.

Since we need to analyze the speed of gravity, so we must first figure out what is the relationship between gravity and velocity?

\section{Derivation of the Relationship between Gravity and Velocity based on Newton's Gravity Equation}

In a very short time slice dt, we can assume that $m$ is stationary and the gravity received is constant. We can then accumulate the impulse generated by the gravity on each time slice and find the average relating to the entire time period to obtain effective constant gravitation and determine the relationship between the equivalent gravitation and velocity.

Consider the influence of velocity on gravity when the moving velocity of object $m$ relative to $M$ is not 0 .

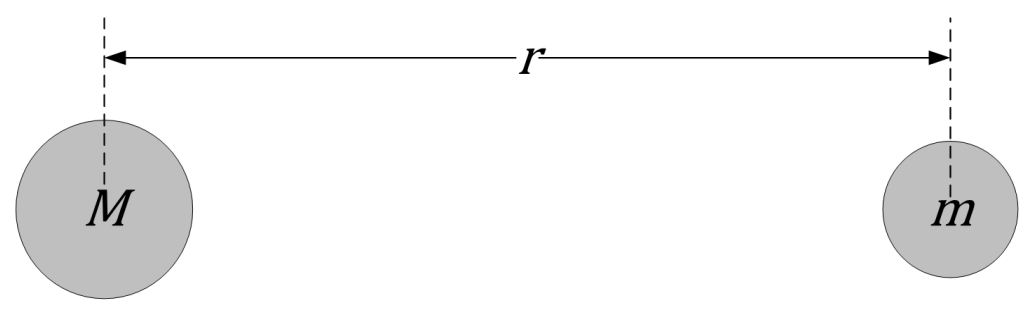

Figure 1: Gravity model

As shown in Figure 1, there are two objects with masses $M$ and $m$, the distance between them is $r, m$ has a moving velocity relative to $M$, the speed is $v$ and the direction of the velocity is depicted by the straight line connecting them. $F(t)=\frac{G_{0} M m}{(r+v t)^{2}}$ represents the gravity on $m$ at time $t$. The Newtonian equation of gravity is used here. In any small time dt, $m$ can be regarded as stationary. An accumulation of the impulse 
$d p$ is obtained by multiplying the gravity and time in these small time slices. Then, the sum of the gravitational impulse received by $m$ within a certain period can be obtained. Supposing that the gravitational impulse obtained by $m$ is $p$ after time $T$ has passed, the gravity is integrated into the time domain:

$$
p=\int_{0}^{T} F(t) \times d t=\int_{0}^{T} \frac{G_{0} M m}{(r+v t)^{2}} \times d t=\frac{G_{0} M m}{r^{2}} \times \int_{0}^{T} \frac{1}{(1+v t / r)^{2}} \times d t,
$$

$p=\frac{G_{0} M m}{r^{2}} \times\left.\frac{-r / v}{1+v t / r}\right|_{0} ^{T}$,

$p=\frac{G_{0} M m}{r^{2}} \times \frac{T}{1+v T / r}$.

For an object $m$ with a speed of $v$, the accumulated impulse $p$ during time $T$ can be expressed by an equivalent constant force multiplied by time $T$. For the convenience of description, we use $F(v)$ to express this equivalent force.

$$
F(v)=p / T=\frac{G_{0} M m}{r^{2}} /\left(1+\frac{v T}{r}\right) .
$$

There is an inverse proportional relationship between the equivalent gravitational force and the speed $v$. The larger the $v$, the smaller the $F(v)$; the smaller the $v$, the larger the $F(v)$. When $v=0$, it is Newtonian gravity. When $v$ tends to infinity, $F(v)=$ 0 . The Newtonian gravitational equation is based on the premise that the gravitational field speed is infinite. Now, we may assume that the gravitational field has a finite speed $X$, therefore, the Newtonian gravitational equation is no longer applicable and need to be modified.

Let us continue to think about the difference in the average gravitational force received by two objects at different speeds during time T? Assuming that two objects have different velocities, $v_{1}=v_{0}-\delta v, v_{2}=v_{0}+\delta v$, their average gravity:

$$
\begin{aligned}
& F\left(v_{1}\right)=F\left(v_{0}-\delta v\right)=\frac{G_{0} M m}{r^{2}} /\left(1+\frac{\left(v_{0}-\delta v\right) T}{r}\right) . \\
& F\left(v_{2}\right)=F\left(v_{0}+\delta v\right)=\frac{G_{0} M m}{r^{2}} /\left(1+\frac{\left(v_{0}+\delta v\right) T}{r}\right) . \\
& F\left(v_{1}\right)-F\left(v_{2}\right)=\frac{G_{0} M m}{r^{2}}\left(\frac{r}{r+\left(v_{0}-\delta v\right) T}-\frac{r}{r+\left(v_{0}+\delta v\right) T}\right) . \\
& F\left(v_{1}\right)-F\left(v_{2}\right)=\frac{G_{0} M m}{r^{2}}\left(\frac{2 r \delta v T}{\left(r+v_{0} T\right)^{2}-(\delta v T)^{2}}\right) .
\end{aligned}
$$

When $T$ is infinitesimal close to $0, F\left(v_{1}\right)-F\left(v_{2}\right)=\frac{G_{0} M m}{r^{2}}\left(\frac{2 \delta v T}{r}\right)$.

Assume $K=\frac{T}{r}, \delta v=v_{1}-v_{2}$, so the following formula is obtained:

$F\left(v_{1}\right)-F\left(v_{2}\right)=\frac{G_{0} M m}{r^{2}}(K \delta v)$, we can see that there is a linear relationship between average gravity and velocity, when $T$ is infinitesimal close to 0 , the average gravity is the instantaneous gravity.

However, we also know that if there is relative velocity between any two objects, there will be a general Doppler effect between them. According to this general Doppler effect 
between the object and the gravitational field, two Doppler effect boundary conditions are introduced:

1. When an object's velocity relative to the source of gravity is 0 , it is Newtonian gravity.

2. When an object's velocity relative to the gravitational field is 0 , the gravitational force no longer acts on the object.

As shown in the Figure 2, according to the general Doppler effect (chase effect), using boundary conditions $F(0)=\frac{G_{0} M m}{r^{2}}$ and $F(X)=0$, it can be easily calculated:

$$
F(v)=F(0)+v \times \frac{F(X)-F(0)}{X}=F(0) \times \frac{X-v}{X}=\frac{G_{0} M m}{r^{2}} \times \frac{X-v}{X} .
$$

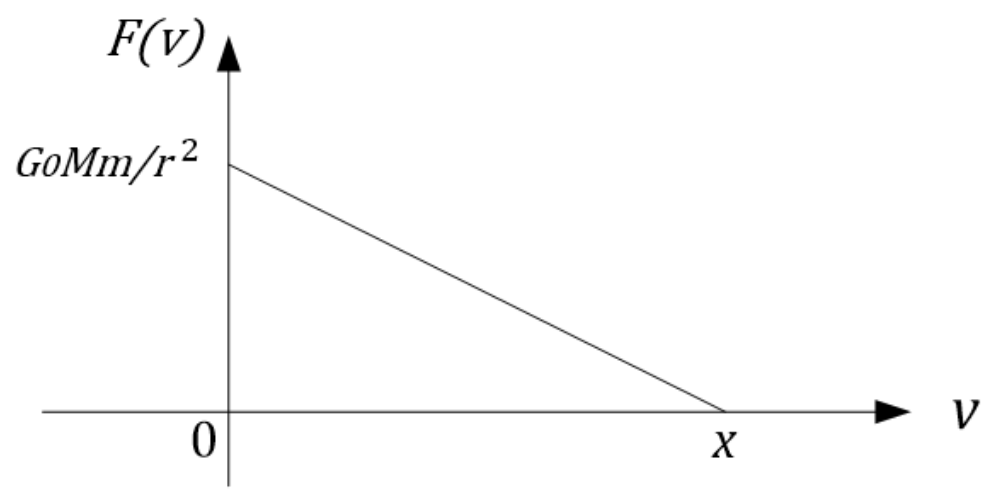

Figure 2: Linear relationship between gravity and velocity

From the above analysis, the formula of universal gravitation with parameter $v$ is as follows:

$$
F(v)=\frac{G_{0} M m}{r^{2}} \times f(v), f(v)=\frac{X-v}{X} .
$$

If it is necessary to preserve the form of Newton's gravity equation, we may write it as follows:

$$
F(v)=G(v) \times \frac{M m}{r^{2}}, G(v)=G_{0} \times \frac{X-v}{X} .
$$

That is, the gravitational constant becomes a function of $v, G(v)$. Thus, we may understand that when the gravitational field has a different speed relative to $m$, the gravitational constant is also different. Next, we apply the new gravitational equation to the planetary orbit calculation to determine whether it is consistent with actual observations. 


\section{Calculation of the Influence of the New Gravitational Equation on Earth's Orbit}

From the above derivation, we get the gravity formula with $v$ as a parameter:

$$
F(v)=\frac{G_{0} M m}{r^{2}} \times \frac{X-v}{X} .
$$

Considering that the velocity direction of the object $m$ may have an angle with the gravitational field, we define $v_{r}$ as the component of the speed in the direction of the gravitational field and then obtain a general formula:

$$
F\left(v_{r}\right)=\frac{G_{0} M m}{r^{2}} \times \frac{X-v_{r}}{X} .
$$

The equation shows that when an object has a velocity component in the direction of the gravitational field, that is, there is a movement effect in the same direction between the gravitational field and the object, the gravitational force received decreases. When the object has a velocity component that is opposite to the direction of the gravitational field, that is, the two have the effect of moving towards each other, the gravitational force received increases. This leads us to thus consider what impact, under this general Doppler effect, it may have on the planet's orbit. Can planets maintain the conservation of mechanical energy in their orbits?

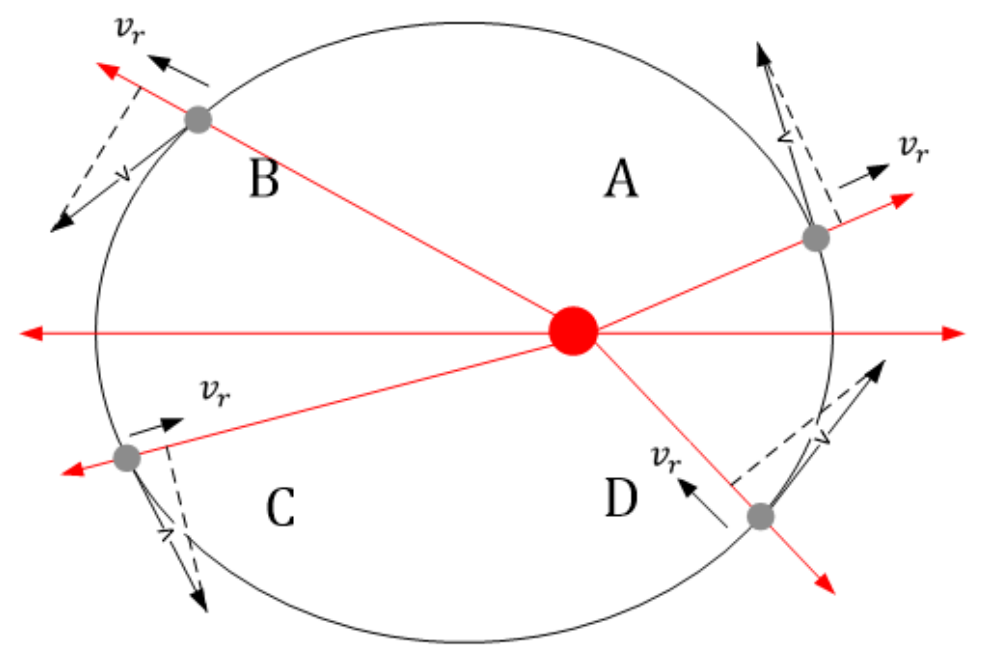

Figure 3: The velocity component of the planet's gravitational field direction

As shown in Figure 3, under the new gravitational equation, as the planetary velocity has the same direction component $v_{r}$ in the direction of the gravitational field in orbits $\mathrm{A}$ and $\mathrm{B}$, the gravity decreases. Therefore, the planet gains extra force in the direction of the gravitational field. This force travels in the same direction as $v_{r}$. According to the power calculation formula $P=F \times v_{r}>0$, the planetary mechanical energy increases.

Regarding regions $\mathrm{C}$ and $\mathrm{D}$, as the planetary velocity has a reverse component $v_{r}$ in the direction of the gravitational field, the gravitational force increases. Therefore, the 
extra force gained by the planet moves in the opposite direction of the gravitational field. This force is in the same direction as $v_{r}$. According to the power calculation formula $P=F \times v_{r}>0$, the planetary mechanical energy increases.

Therefore, under the new gravitational equation, the mechanical energy of the planet in the entire orbit continues to increase and the mechanical energy becomes larger and larger. This would cause the planet to gradually move away from the Sun and eventually the solar system. Taking Earth as an example, using the new gravitational equation, after how many revolution cycles would Earth begin to move away from the solar system? Below we include our theoretical analysis and calculations.

\subsection{Introduction of Polar Coordinates}

Let the Sun, mass $M$, lie at the origin. Consider a planet, mass $m$, in orbit around the Sun. Let the planetary orbit lie in the $x-y$ plane. Let $\mathbf{r}(t)$ be the planet's position vector with respect to the Sun. The planet's equation of motion is

$$
m \ddot{\mathbf{r}}=-\frac{G_{0} M m}{r^{2}} \times \frac{X-v}{X} \times e_{r}
$$

where $e_{r}=\mathbf{r} / r$ and $v_{r}=e_{r}$. . Let $r=|\mathbf{r}|$ and $\theta=\tan ^{-1}(y / x)$ be plane polar coordinates. The radial and tangential components of (4) are

$$
\begin{gathered}
\ddot{r}-r \dot{\theta}^{2}=-\frac{G_{0} M}{r^{2}}\left(1-\frac{\dot{r}}{X}\right) \\
r \ddot{\theta}+2 \dot{r} \dot{\theta}=0
\end{gathered}
$$

(6) can be integrated to give

$$
r^{2} \dot{\theta}=h
$$

where $\mathrm{h}$ is the conserved angular momentum per unit mass. (5),(7) can be combined to give

$$
\ddot{r}-\frac{h^{2}}{r^{3}}=-\frac{G_{0} M}{r^{2}}\left(1-\frac{\dot{r}}{X}\right)
$$

\subsection{Energy Conservation}

Multiply (8) by $\dot{r}$. We obtain

$$
\frac{d}{d t}\left(\frac{\dot{r}^{2}}{2}+\frac{h^{2}}{2 r^{2}}-\frac{G_{0} M}{r}\right)=\frac{G_{0} M \dot{r}^{2}}{r^{2} X}
$$


or

$$
\frac{d \epsilon}{d t}=\frac{G_{0} M \dot{r}^{2}}{r^{2} X} \geq 0
$$

where

$$
\epsilon=\frac{1}{2}\left(\dot{r}^{2}+r^{2} \dot{\theta}^{2}\right)-\frac{G_{0} M}{r}
$$

is the energy per unit mass. (10) demonstrates that the Doppler shift correction to the law of force causes the system to cease conserving energy. The orbital energy grows without limit. This means that the planet will eventually escape the Sun's gravitational pull (when its orbital energy becomes positive).

\subsection{Solution of Equations of Motion}

Let $1 / r=u[\theta(t)]$. It follows that

$$
\begin{gathered}
\dot{r}=-h \frac{d u}{d \theta}, \\
\ddot{r}=-u^{2} h^{2} \frac{d^{2} u}{d \theta^{2}},
\end{gathered}
$$

thus, Eq. (8) becomes

$$
\frac{d^{2} u}{d \theta^{2}}-\gamma \frac{d u}{d \theta}+u=\frac{G_{0} M}{h^{2}}
$$

where

$$
\gamma=\frac{G_{0} M}{h X}
$$

is a small dimensionless constant. To first order in $\gamma$, an appropriate solution of (14) is

$$
u \approx \frac{G_{0} M}{h^{2}}(1+e \exp (\gamma \theta) \cos \theta),
$$

where $e$ is the initial eccentricity of the orbit. Thus

$$
r(\theta)=\frac{r_{c}}{1+e \exp (\gamma \theta) \cos \theta},
$$


where

$$
r_{c}=\frac{h^{2}}{G_{0} M} .
$$

It can be observed that the orbital eccentricity grows without limit as the planet orbits the Sun. Eventually, when the eccentricity becomes unity, the planet will escape the Sun.

\subsection{Estimation of Escape Time}

The planet escapes when its orbital eccentricity becomes unity. The number of orbital revolutions, $n$, required for this to happen is

$$
e \exp (\gamma n 2 \pi)=1
$$

where $e$ is the initial eccentricity. Thus, $n=\frac{1}{2 \pi \gamma} \ln \left(\frac{1}{e}\right)$,

$$
\gamma=\frac{2 \pi a}{T X\left(1-e^{2}\right)^{\frac{1}{2}}}
$$

where a is the initial orbital major radius and $T$ is the initial period. Hence,

$$
n=\frac{T X\left(1-e^{2}\right)^{\frac{1}{2}} \ln \left(\frac{1}{e}\right)}{4 \pi^{2} a} .
$$

For Earth, $T=3.156 \times 10^{7} \mathrm{~s}, X=c=2.998 \times 10^{8} \mathrm{~m} / \mathrm{s}, a=1.496 \times 10^{11} \mathrm{~m}$, and $e=0.0167$. Hence, Earth would escape from the Sun's gravitational influence after

$$
n=\frac{\left(3.156 \times 10^{7}\right)\left(2.998 \times 10^{8}\right)\left(1-0.0167^{2}\right)^{\frac{1}{2}} \ln \left(\frac{1}{0.0167}\right)}{4 \pi^{2}\left(1.496 \times 10^{11}\right)} \approx 6.6 \times 10^{3}
$$

revolutions. If each revolution takes approximately 1 year, then the escape time is a few thousand years. However, the age of the solar system is $4.6 \times 10^{9}$ years. The escape time is smaller than this by a factor of approximately one million. Therefore, the speed of gravitational waves cannot represent the speed of the gravitational field. From equation (22), the speed of a gravitational field $X$ must be much greater than the speed of light $c^{[10]}$; this is more in line with Newton's argument that the force of gravity acts at a distance.

We may consider the following analogy: we use a rope to pull a kite. When we shake it hard, the rope will fluctuate and pass to the kite at a certain wave speed, however, 
when we loosen the rope, the kite instantly loses control. It is inappropriate to use the wave speed of the rope to represent the speed of the force of the rope on the kite. With this considered, how do gravitational waves affect gravity? Since the revolution speed of the Sun will cause gravitational waves, how are gravitational waves distributed around the Sun?

\section{The Influence of Gravitational Waves Produced by the Sun on the Surrounding Gravity}

Gravitational waves caused by the movement of the Sun are akin to water waves caused by ships. For the convenience of explanation, we have turned the three-dimensional space problem into a two-dimensional problem. The gravitational influence caused by gravitational waves is different in the direction of the Sun's velocity and the vertical direction, as shown in Figure 4.

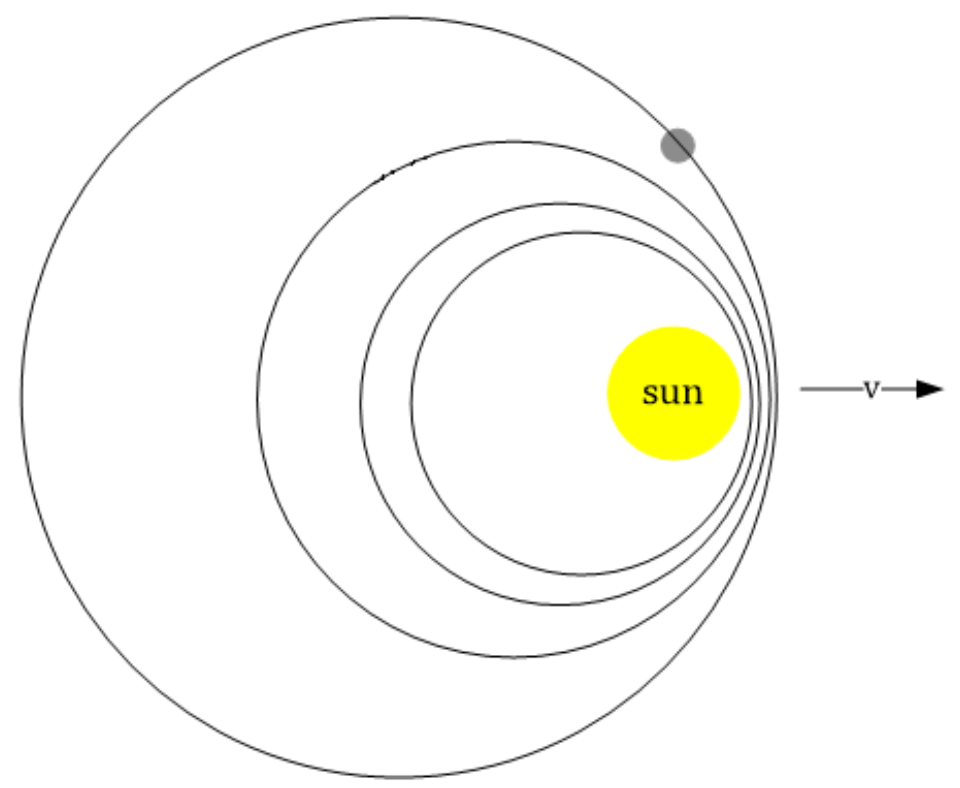

Figure 4: The gravitational wave model generated by the Sun's movement.

Assuming that, without considering the general Doppler effect, the ratio of the gravitational increase caused by gravitational waves to Newtonian gravitation is $r_{w}$, we introduce a gravitational wave influence factor of $f_{w}$. Figure 4 shows that, due to the general Doppler effect of gravitational waves, the energy of gravitational waves is largest in the direction of the Sun's velocity and the impact on gravity is the greatest. The planet's orbital surface is perpendicular to the direction of the Sun's velocity and the gravitational wave is relatively small. 


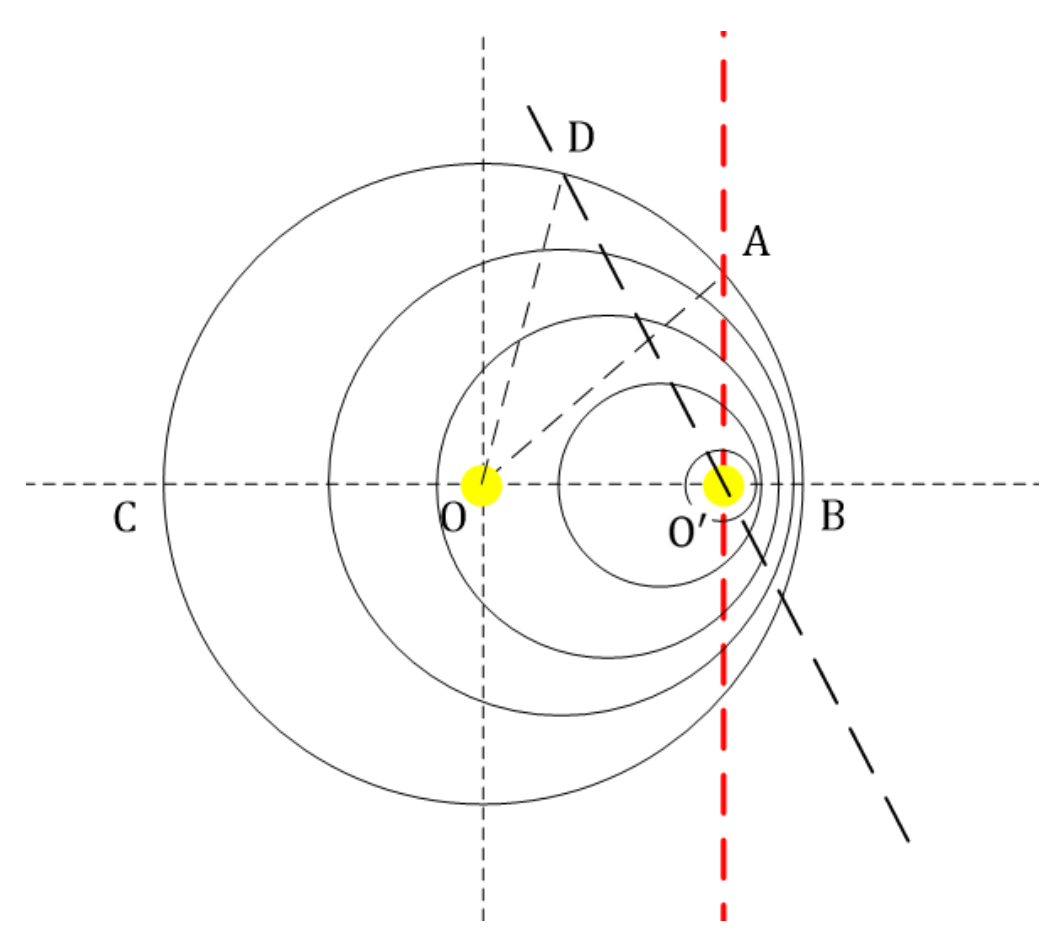

Figure 5: The solar gravitational wave calculation model

\subsection{Calculation of the Influence Factor of Gravitational Waves in the Direction of the Sun's Velocity}

We know that the revolution speed of the Sun is $v_{s}$. Assuming that the Sun moves from position $O$ to position $O^{\prime}$ after time $T$, the gravitational waves generated in the direction of the Sun's velocity during this period are all located between $O^{\prime} B$. According to the general Doppler effect of gravitational waves, the influence factor of gravitational waves in this direction is as below:

$$
f_{w}=\frac{c+v_{s}}{c}>1.0 .
$$

\subsection{Calculation of the Influence Factor of Gravitational Waves in the Vertical Direc- tion of the Sun's Velocity}

The gravitational waves in the direction perpendicular to the Sun's velocity are located between $O^{\prime} A$; it is only necessary to calculate the ratio between $O^{\prime} B$ and $O^{\prime} A$ to determine the gravitational wave density relationship in the two directions.

$$
O^{\prime} B=c T-v_{s} T
$$




$$
O^{\prime} A=\left[(c T)^{2}-\left(v_{s} T\right)^{2}\right]^{\frac{1}{2}},
$$

thus:

$$
f_{w} \approx \frac{c+v_{s}}{c} \times\left(\frac{c-v_{s}}{c+v_{s}}\right)^{\frac{1}{2}}=\left(\frac{c^{2}-v_{s}^{2}}{c^{2}}\right)^{\frac{1}{2}}
$$

Substituting the solar revolution speed $v_{s}=240 \times 10^{3} \mathrm{~m} / \mathrm{s}$ and the gravitational wave speed $c=2.998 \times 10^{8} \mathrm{~m} / \mathrm{s}$, we get $\frac{O^{\prime} B}{O^{\prime} A}=\left(\frac{c-v_{s}}{c+v_{s}}\right)^{\frac{1}{2}} \approx 0.9992$. Figure 4 shows that the density of gravitational waves in the vertical direction is smaller than that in the direction of the Sun's velocity. The density of gravitational waves gradually decreases from the direction of the Sun's velocity to the vertical direction. If the gravitational wave density is equivalent to the level of the depression in the plane, then this gravitational wave density model is somewhat similar to the space-time depression model described by general relativity (GR). As shown in the Figure 6, the gravitational wave density presents a non-uniform distribution; gravitational waves have the highest density in the direction of the sun's velocity (bottom of Figure 6), and gradually decrease upwards.

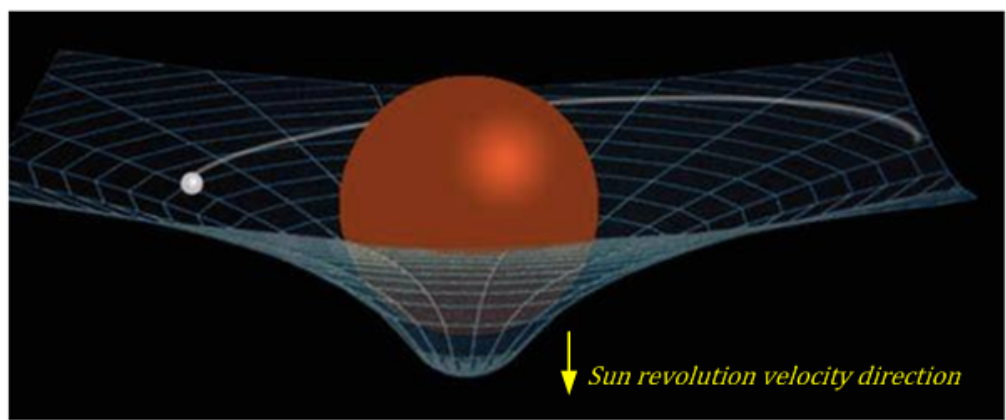

Figure 6: Gravitational wave density model

\subsection{Calculation of the Influence Factor of Gravitational Waves on the Planetary Or- bital Surface}

We know that the planet's orbital plane is approximately perpendicular to the direction of the Sun's motion; thus, the red line in Figure 5 represents the ideal orbital plane of the planet (completely perpendicular to the direction of the sun's velocity). According to formula (26), we can calculate the influence factor of gravitational waves on the orbital surface and thus determine that this value will be less than 1.0. 
4.4 Calculation of the Influence Factor of Gravitational Waves on the Reverse of the Sun's Velocity

Behind the vertical plane (to the left of the red line in Figure 5), shows that the density of the gravitational waves will continue to decrease and reach a minimum in the opposite direction of the Sun's velocity. At this time $\frac{O^{\prime} B}{O^{\prime} C}=\frac{c-v_{s}}{c+v_{s}}$, the gravitational wave influence factor is as below:

$$
f_{w} \approx \frac{c+v_{s}}{c} \times \frac{c-v_{s}}{c+v_{s}}=\frac{c-v_{s}}{c} .
$$

Substituting $v_{s}=c$ into (26) and (27), it can be determined that when the speed of the Sun reaches $c$, the orbital surface of the planet perpendicular to the Sun's velocity (the position of the red line) and the position behind it (the left side of the red line) is no longer affected by gravitational waves.

\subsection{Calculation of the Influence Factor of Gravitational Waves at any Position}

As shown in Figure 5, assuming that the angle between $O^{\prime} D$ and the red line is $\theta$ (with $D$ at any position), then

$$
O D^{2}=O^{\prime} D^{2}+O O^{\prime 2}-2 O^{\prime} D \times O O^{\prime} \cos \left(\frac{\pi}{2}-\theta\right)
$$

we get:

$$
O^{\prime} D=\frac{2 O O^{\prime} \cos \left(\frac{\pi}{2}-\theta\right)+\left[4\left(O O^{\prime} \cos \left(\frac{\pi}{2}-\theta\right)\right)^{2}-4\left(O O^{\prime 2}-O D^{2}\right)\right]^{\frac{1}{2}}}{2},
$$
then,

$$
\frac{O^{\prime} B}{O^{\prime} D}=\frac{2 O^{\prime} B}{2 O O^{\prime} \cos \left(\frac{\pi}{2}-\theta\right)+\left[4\left(O O^{\prime} \cos \left(\frac{\pi}{2}-\theta\right)\right)^{2}-4\left(O O^{\prime 2}-O D^{2}\right)\right]^{\frac{1}{2}}}
$$

thus:

$$
f_{w} \approx\left(\frac{c+v_{s}}{c}\right) \times \frac{c-v_{s}}{v_{s} \cos \left(\frac{\pi}{2}-\theta\right)+\left[\left(v_{s} \cos \left(\frac{\pi}{2}-\theta\right)\right)^{2}-\left(v_{s}^{2}-c^{2}\right)\right]^{\frac{1}{2}}} .
$$

\subsection{The Influence of Gravitational Waves on Gravity}

Assuming that the gravitational force of an object under the influence of gravitational waves is $F_{w}, F_{w}$ can be regarded as two parts:

Part 1: Newtonian gravity $F=\frac{G_{0} M m}{r^{2}}$.

Part 2: The gravity contributed by the gravitational wave $r_{w} f_{w} F$, where $r_{w}$ is the ratio of the gravitational increase caused by gravitational waves to Newtonian gravitation. 
Thus, we get:

$$
F_{w}=F+r_{w} \times f_{w} \times F
$$

Let us take the orbital position as an example to illustrate the calculation of gravity under the influence of gravitational waves:

$$
F_{w}=F+r_{w} \times f_{w} \times F=F \times\left(1+r_{w} \times\left(\frac{c^{2}-v_{s}^{2}}{c^{2}}\right)^{\frac{1}{2}}\right) .
$$

As there is also a general Doppler effect between planets and gravitational waves, it is also necessary to consider the influence of this factor. Assuming that the speed of the planet is $v_{p}$ and the speed of the planet in the direction of the gravitational wave is $v_{p w}$, then the chase factor $\frac{c-v_{p w}}{c}$ between the planet and the gravitational wave can be obtained and this factor is put into (32) to get:

$$
F_{w}=F \times\left(1+r_{w} \times\left(\frac{c^{2}-v_{s}^{2}}{c^{2}}\right)^{\frac{1}{2}} \times \frac{c-v_{p w}}{c}\right)
$$

substituting $F$, we get:

$$
F_{w}=\frac{G_{0} M m}{r^{2}} \times\left(1+r_{w} \times\left(\frac{c^{2}-v_{s}^{2}}{c^{2}}\right)^{\frac{1}{2}} \times \frac{c-v_{p w}}{c}\right)
$$

here $r_{w} \approx 0.00058$; this value was derived from a program simulation.

In the same way, the gravity of other positions can be calculated. We write the gravity equation of any position:

$$
F_{w}=\frac{G_{0} M m}{r^{2}} \times\left(1+r_{w} \times \frac{c+v_{s}}{c} \times\left(\frac{c-v_{s}}{v_{s} \cos \left(\frac{\pi}{2}-\theta\right)+\left[\left(v_{s} \cos \left(\frac{\pi}{2}-\theta\right)\right)^{2}-\left(v_{s}^{2}-c^{2}\right)\right]^{\frac{1}{2}}} \times \frac{c-v_{p w}}{c}\right)\right)
$$

\subsection{Gravitational Waves Caused by the Rotation of the Sun}

The Sun's rotation can also cause gravitational waves, however, the Sun's revolution speed of $240 \mathrm{~km} / \mathrm{s}$ is much greater than its rotation speed of $2 \mathrm{~km} / \mathrm{s}$. As such, this physical model does not consider the influence of gravitational waves caused by rotation. To obtain more precise calculations, we must consider this factor. 


\section{Analysis of the Influence of Gravitational Waves on Planetary Orbits}

If the planet's orbital surface is not completely perpendicular to the velocity of the Sun and the orbit is split over both sides of the red line, then the impact of gravitational waves on planets is also irregular, which affects the orbit and contributes part of the force to planetary precession ${ }^{[9]}$. The closer the planet's orbit is to the Sun, the greater the gravitational wave density gradient and the more obvious the effect of precession; the farther the distance, the less obvious. Similarly, the larger the angle between the real planetary orbit surface and the red line in Figure 5, the more obvious the precession.

In 1915, Albert Einstein published in $[1915 \text {, p. 839 }]^{[9]}$ a formula for the relativistic perihelion shift, for one period, of

$$
\varepsilon=24 \pi^{3} \frac{a^{2}}{T^{2} c^{2}\left(1-e^{2}\right)},
$$

where according to contemporary data $T$ is the orbital period of planet, $e$ is the eccentricity of its elliptical orbit, $a$ is the length of its corresponding semimajor axis, and $c$ is the speed of light in vacuum.

$$
\delta \dot{\varphi}=\varepsilon \frac{\tau}{T} \frac{180}{\pi} 3600 "
$$

here $\tau=3155814954 \mathrm{~s}$ is the number of seconds in one century. We can also use a simplified calculation formula of GR.

$$
\delta \dot{\varphi} \simeq \frac{0.0383}{R T} .
$$

From the formulas (37) and (38), GR does not consider the angle between the real planet's orbital plane and the Sun's vertical plane (the red line in Figure 5), and the eccentricity of the orbit is not the main factor either, when calculating the planetary precession. However, we must consider them as the main factors in the data calculated by formula (35). These may be the biggest differences between the two. Below, we substitute the $R$ and $T$ values of each planet (see Figure 7) for GR calculation. 


\begin{tabular}{||l||l|l||l||}
\hline Planet & $\mathrm{M} / \mathrm{M}_{0}$ & $\mathrm{~T}(\mathrm{yr})$ & $\mathrm{R}(\mathrm{au})$ \\
\hline \hline Mercury & $1.66 \times 10^{-7}$ & 0.241 & 0.387 \\
\hline \hline Venus & $2.45 \times 10^{-6}$ & 0.615 & 0.723 \\
\hline \hline Earth & $3.04 \times 10^{-6}$ & 1.000 & 1.00 \\
\hline \hline Mars & $3.23 \times 10^{-7}$ & 1.881 & 1.52 \\
\hline \hline Jupiter & $9.55 \times 10^{-4}$ & 11.86 & 5.20 \\
\hline \hline Saturn & $2.86 \times 10^{-4}$ & 29.46 & 9.54 \\
\hline \hline Uranus & $4.36 \times 10^{-5}$ & 84.01 & 19.19 \\
\hline \hline Neptune & $5.18 \times 10^{-5}$ & 164.8 & 30.07 \\
\hline \hline
\end{tabular}

Figure 7: Data for the major planets in the solar system, giving the planetary mass relative to that of the Sun, the orbital period in years, and the mean orbital radius relative to that of Earth.

The calculated precession data of each planet per century is as follows:

Mercury 41.06"

Venus 8.6"

Earth $3.83 "$

Mars 1.34"

Jupiter 0.062"

Saturn 0.0136"

Uranus $0.00238 "$

But we must note that when GR calculates the planet precession deviation, it ignores the rotation of the Sun around the center of mass of the solar system and the influence of planets on the Sun's gravity. GR constructs an idealized 1-body model. 1-body means there is only one planet in the solar system.

In order to maintain consistency with GR, we also made the same omission, constructed the same 1-body ideal model, and calculated the precession of each planet. If we want the calculated results to be closer to the real 1-body system, we cannot ignore the influence of the planets on the Sun, nor the rotation of the Sun around the center of mass of the 1-body system. We have made a clear comparison of all calculated data in the table below. We can see that the gravitational model constructed according to formula (35), without considering the influence of gravitational waves (that is, classical Newtonian mechanics), the planet precession is zero. And considering the influence of gravitational waves, the planet precession in the 1-body system is relatively close to the results calculated by GR. We did not find the data of GR in the real 1-body system, but 
according to the analysis of GR, the changes in the data are very small. The data we calculated using the gravitational wave theory also reflected this.(The precession data in the paper are all calculated after the perihelion is projected onto the $\mathrm{x}-\mathrm{y}$ plane.)

\begin{tabular}{|l|l|l|l|l|l|l|}
\hline Condition & Planet & \multicolumn{2}{|c|}{ Real 1-body model } & \multicolumn{2}{c|}{ Ideal 1-body model } \\
\cline { 3 - 8 } & & $\begin{array}{c}\text { Gravity } \\
\text { wave ON }\end{array}$ & $\begin{array}{c}\text { Gravity } \\
\text { wave OFF }\end{array}$ & $\begin{array}{c}\text { Gravity } \\
\text { wave ON }\end{array}$ & $\begin{array}{c}\text { Gravity } \\
\text { wave OFF }\end{array}$ & GR \\
\hline \multirow{2}{*}{$\begin{array}{c}\text { 1. 1-Body. } \\
\text { 2. Time accuracy } \\
\text { is 0.2s. }\end{array}$} & Mercury & 43 & 0 & 43 & 0 & 41.06 \\
\cline { 2 - 8 } & Venus & 169 & 0 & 169 & 0 & 8.6 \\
\cline { 2 - 8 } $\begin{array}{c}\text { 3. The unit is arc } \\
\text { seconds. }\end{array}$ & Earth & 0.35 & 0 & 0.35 & 0 & 3.83 \\
\cline { 2 - 8 } & Mars & -5.6 & 0 & -5.6 & 0 & 1.34 \\
\cline { 2 - 8 } & Jupiter & -1.35 & 0 & -1.35 & 0 & 0.062 \\
\cline { 2 - 8 } & Saturn & -0.33 & 0 & -0.33 & 0 & 0.0136 \\
\cline { 2 - 8 } & Uranus & 0.1 & 0 & 0.1 & 0 & 0.00238 \\
\cline { 2 - 7 } & Neptune & -0.64 & 0 & -0.64 & 0 & \\
\hline
\end{tabular}

Figure 8: 1-body planetary orbit precession per century

Except for Venus's precession data of 169 " vs 8.6 ", the data of other planets are relatively close to GR.

Let us examine the characteristics of Venus: Venus's eccentricity is abnormally low $(e=0.0068)$, which makes its perihelion extremely sensitive to small disturbances. However, the angle between its orbit and the vertical plane of the Sun is very large $\left(3.39^{\circ}\right)$; thus, we have reason to believe that gravitational waves will have a significant influence on the orbital precession of Venus.

Why is the data of Venus (169" vs 8.6") so different? From formulas (37) and (38), it can be determined that GR does not take eccentricity as the main factor and does not consider the angle between the orbital surface and the vertical surface of the Sun. Under different eccentricities and angles, the precession data calculated by GR remains the same. This may be the reason for the large difference between the two.

We know that the famous Mercury Precession 43" comes from the comparison between the calculated data of the planetary orbit of the solar system by Newton's classical mechanics and the astronomical observation data. This requires the calculation of all the planets in the solar system, the gravitational force between the planets and the Sun, the gravitational force between the planets, and the rotation of the Sun around the center of mass of the solar system to construct a real N-body system. Then it is necessary to calculate the planet precession data under and without the influence of gravitational waves. Since GR does not provide planetary precession data under the N-body system, it cannot be compared with GR. We can see that the data under the action of gravitational waves are different. For Mercury, the difference between the two is close to the data under the 1-body model 43". (The initial coordinates (x, y, z) and initial velocity (vx, vy, vz) datas of the planets and the Sun used in this paper are all from NASA's 
Horizons System https://ssd.jpl.nasa.gov/horizons/.)

\begin{tabular}{|l|l|l|l|l|l|}
\hline Condition & Planet & Gravity wave OFF & Gravity wave ON & NASA & GR \\
\hline $\begin{array}{l}\text { 1. N-Body. } \\
\begin{array}{l}\text { 2. Time accuracy is } \\
\text { o.2s. }\end{array}\end{array}$ & Mercury & 531 & 572 & 575 & $?$ \\
\cline { 2 - 6 } $\begin{array}{l}\text { 3. The unit is arc } \\
\text { seconds. }\end{array}$ & Venus & $-200 \sim-40$ & $400 \sim 550$ & 204 & $?$ \\
\cline { 2 - 7 } & Earth & $1080 \sim 1180$ & $1080 \sim 1180$ & 1145 & $?$ \\
\cline { 2 - 7 } & Mars & 1590 & 1590 & 1628 & $?$ \\
\cline { 2 - 7 } & Jupiter & $600 \sim 1000$ & $600 \sim 1000$ & 655 & $?$ \\
\cline { 2 - 7 } & Saturn & $1600 \sim 2200$ & $1600 \sim 2200$ & 1950 & $?$ \\
\cline { 2 - 7 } & Uranus & $140 \sim 600$ & $140 \sim 600$ & 334 & $?$ \\
\cline { 2 - 6 } & Neptune & & & 36 & $?$ \\
\hline
\end{tabular}

Figure 9: N-Body planetary orbit precession per century

In addition, we must emphasize that the common period of the orbits of the eight planets in the solar system is very huge, so it is difficult for us to obtain the orbital precession laws of planets with very small eccentricities through short-term calculations. Through 200 years of astronomical observations, we also cannot get the periodic precession laws of all planets, and it takes longer to observe. But for Mercury and Mars, their eccentricity is relatively large, and we can easily get their approximate general laws through calculations or astronomical observations.

Since the orbital data is obtained through integration in the time domain, the averaged precession data obtained in each orbital period has a certain range of variation. The data in the following table is a piece of data randomly selected after 4000 Mercury cycles. We can see that the precession data is changing. As time increases, this change will be further statistically averaged and gradually reduced. We can see that the influence of gravitational waves on Mercury's precession also changes around 39". 


\begin{tabular}{|l|l|l|l|l|}
\hline Condition & Mercury cycles & Gravity wave OFF & Gravity wave ON & Deviation \\
\hline \multirow{2}{*}{$\begin{array}{l}\text { 1. N-Body. } \\
\text { 2. Time accuracy is } \\
\text { 0.2s. }\end{array}$} & 4850 & 532.87 & 572.28 & 39.41 \\
\cline { 2 - 5 } $\begin{array}{c}\text { 3. The unit is arc } \\
\text { seconds. }\end{array}$ & 4851 & 533.21 & 572.64 & 39.43 \\
\cline { 2 - 5 } & 4852 & 532.62 & 572.14 & 39.52 \\
\cline { 2 - 5 } & 4853 & 532.35 & 571.96 & 39.61 \\
\cline { 2 - 5 } & 4854 & 532.05 & 571.55 & 39.5 \\
\cline { 2 - 5 } & 4855 & 532.92 & 572.05 & 39.13 \\
\cline { 2 - 5 } & 4856 & 532.78 & 571.88 & 39.1 \\
\cline { 2 - 5 } & 4857 & 532.23 & 571.50 & 39.27 \\
\cline { 2 - 5 } & 4858 & 531.42 & 570.69 & 39.27 \\
\cline { 2 - 5 } & 4859 & 531.34 & 570.60 & 39.26 \\
\cline { 2 - 5 } & 4860 & 532.39 & 571.36 & 39.18 \\
\cline { 2 - 5 } & 4861 & 532.45 & 571.63 & 39.12 \\
\cline { 2 - 5 } & 4862 & 531.83 & 570.95 & 39.11 \\
\cline { 2 - 5 } & 4863 & 531.32 & 570.55 & \\
\cline { 2 - 5 } & 4864 & 531.51 & 570.62 & \\
\cline { 2 - 5 } & 4865 & 532.79 & & \\
\hline
\end{tabular}

Figure 10: Mercury precession data per century

In addition to causing planetary precession, gravitational waves also cause planets to move away from the Sun. We know there is also a general Doppler effect between the planet's revolution velocity and the gravitational waves caused by the Sun. The previous 3.2 "Energy Conservation" has analyzed the influence of the general Doppler effect on orbital energy. Gravitational waves also cause the planetary orbital mechanical energy to continue to increase; this causes planets to gradually move away from the Sun.

We applied this gravitational theory to calculate the detailed planetary orbit data $(\mathrm{x}, \mathrm{y}, \mathrm{z})$, and used 3D technology to draw these data, as shown in Figure 11 and Figure 12 , we can clearly observe the orbits of the Sun and planets around the center of mass of the solar system. As shown in Figure 13, when we magnify the z-axis data by 10 times, we can clearly see the angle between the planetary orbital surfaces. 


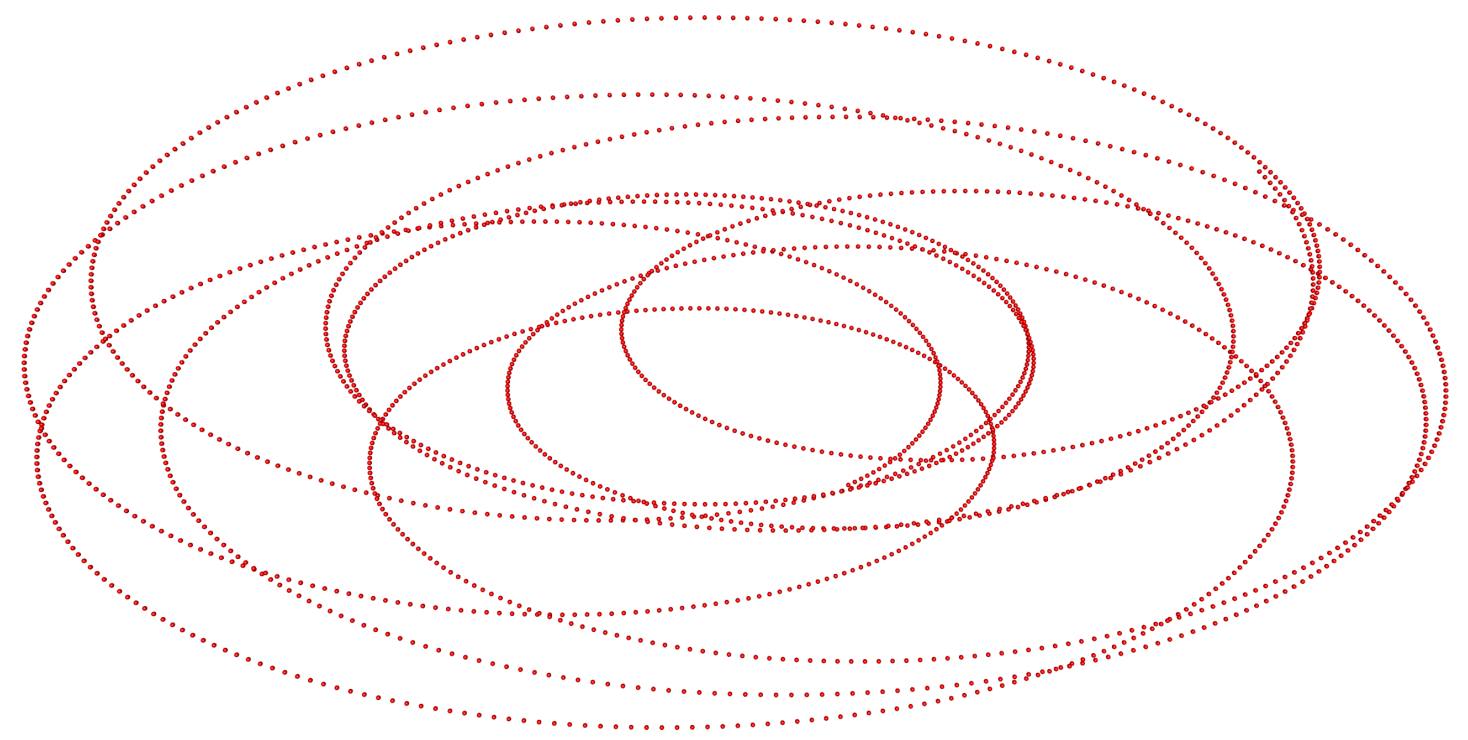

Figure 11: Sun rotation orbit

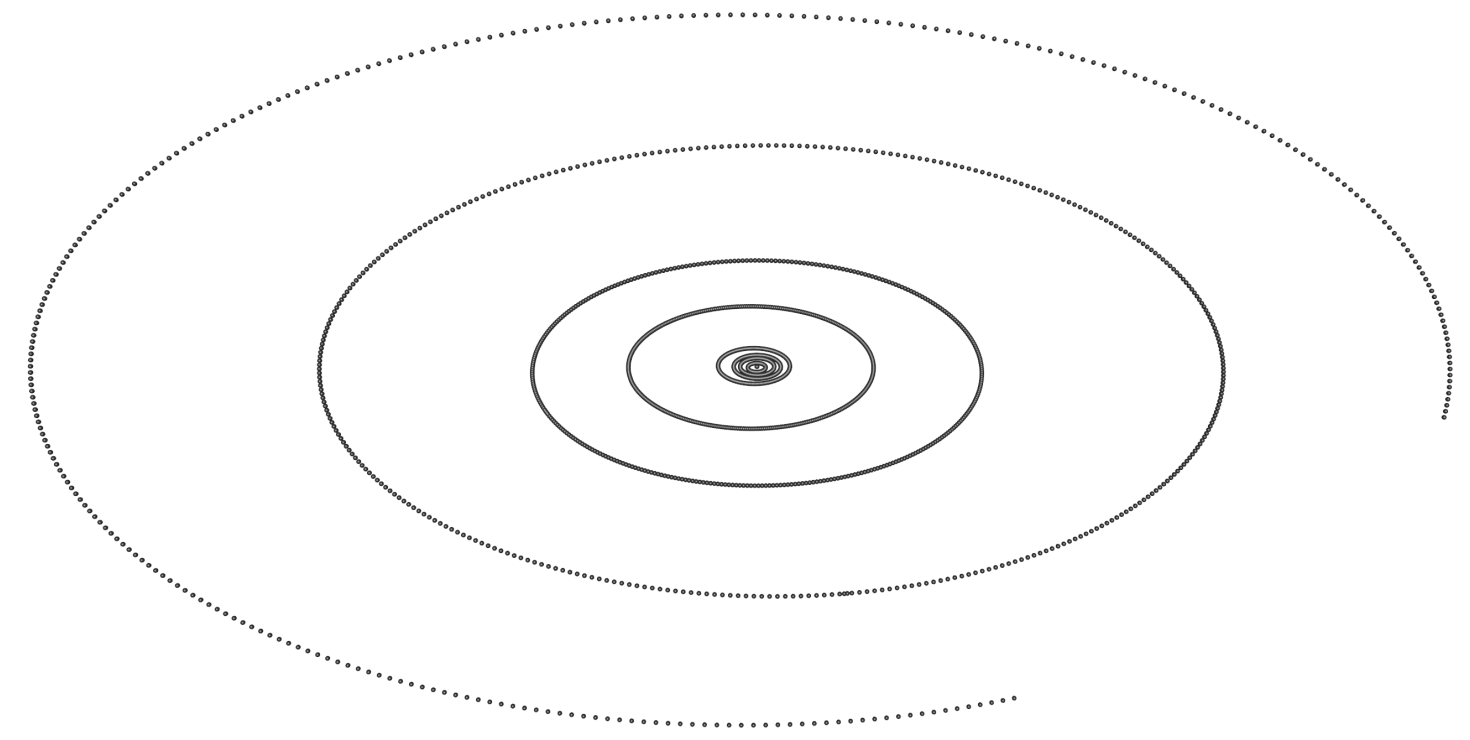

Figure 12: Planetary orbit 


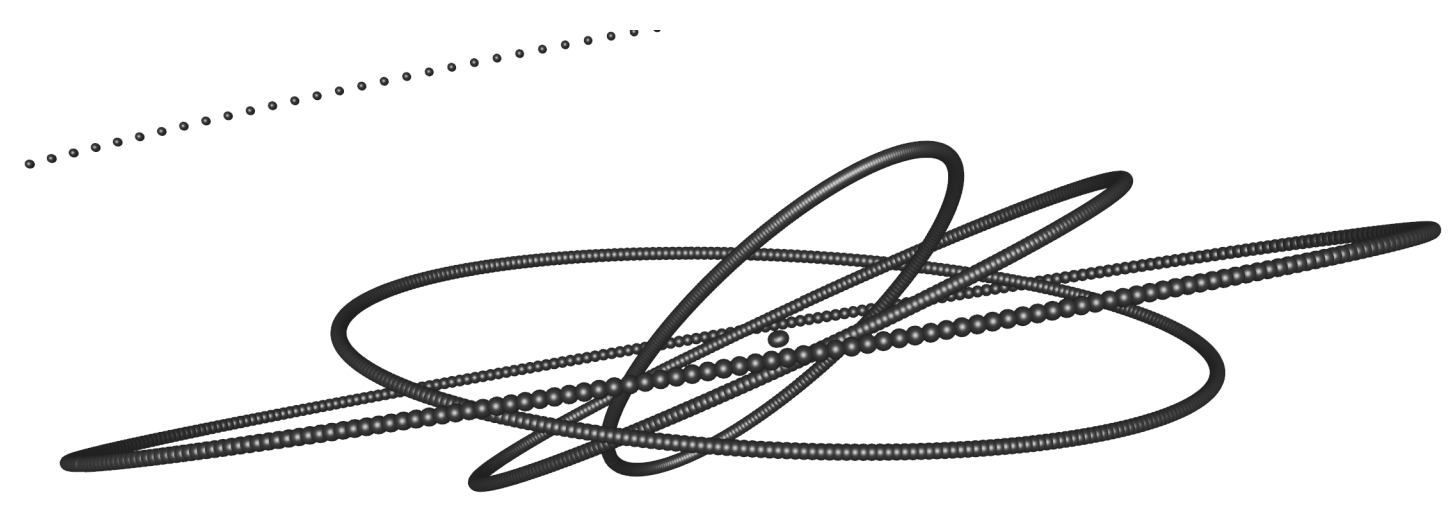

Figure 13: The angle between the planetary orbital surfaces

GR believes that the speed of gravity is equal to the speed of light. If this is true, then the orbits of the planets and the Sun in the solar system should at least remain stable. We have added the gravitational speed parameter to the program, which can be set arbitrarily, such as equal to $0.1 \mathrm{c}$, equal to c, equal to 100c, and so on. Especially in a binary star system (1-body), when the gravitational speed parameter is set to $c$, we can clearly observe that the orbits of the planets and the Sun will no longer be stable. As shown in Figure 14(a), the solar orbit will spiral in a certain direction. By simulating different gravitational speeds, we have come to the conclusion that the lower the gravitational speed, the more unstable the solar system. The theoretical basis of GR is that the speed of gravity is equal to the speed of light, but the accuracy of the orbit simulation is not reflected. Even if the space-time is curved, the orbit will not remain stable.

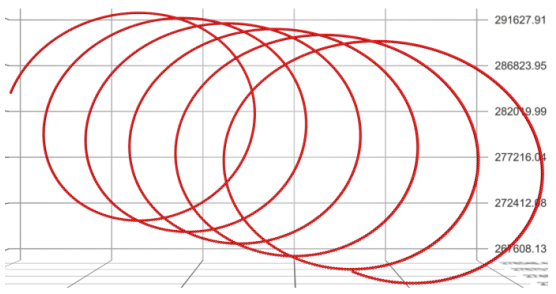

(a) gravitational speed $=\mathrm{c}$

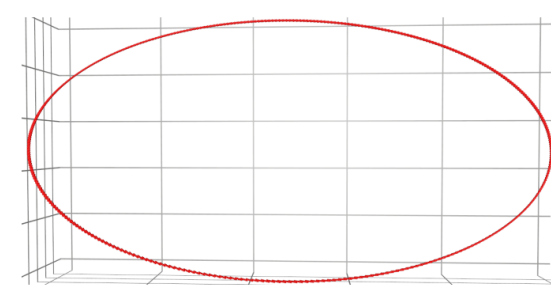

(b) gravitational speed $=1000000 \mathrm{c}$

Figure 14: The orbit of the Sun at different gravitational speeds.

As shown in Figure 15, we have built a very simple Sun-Earth model. If the gravitational speed is equal to the speed of light, the gravitational force of the Sun will be delayed for 8 minutes, then the gravitational force of the Sun on the Earth will come from S1 instead of the true position S0, so there will be a component $F 1$ that is opposite to the orbital velocity of the Sun, under the action of $F 1$, The Earth will move to the left, as shown in Figure 15(a). Finally, the Earth will follow S1, as shown in Figure 
15(b), which is inconsistent with the real solar system. Tom van Flandern's paper ${ }^{[11]}$ also elaborated that the gravitational speed is much greater than the speed of light.

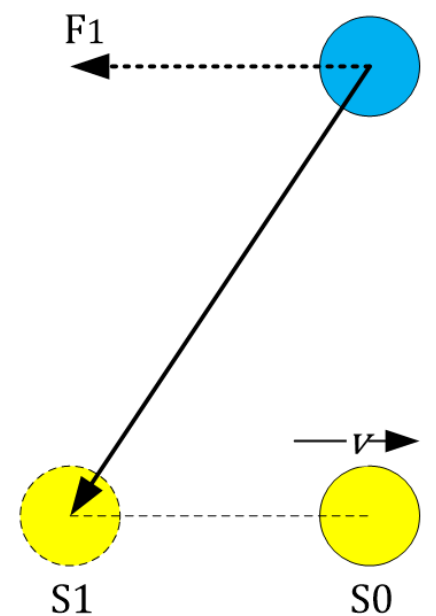

(a)

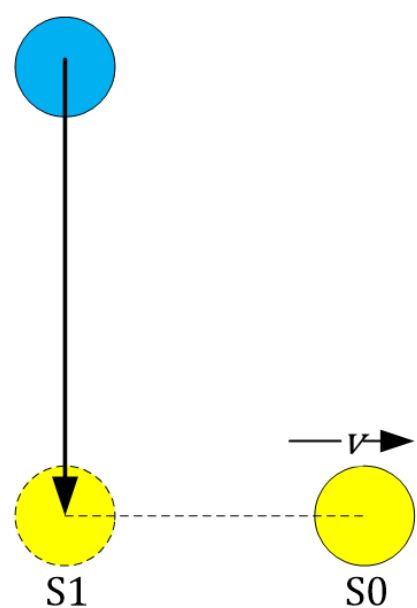

(b)

Figure 15: Sun-Earth model at the gravitational speed equal to the speed of light

\section{Einstein-Infeld-Hoffmann Equations}

The Einstein-Infeld-Hoffmann equations ${ }^{[12]}$ of motion, jointly derived by Albert Einstein, Leopold Infeld and Banesh Hoffmann, are the differential equations of motion describing the approximate dynamics of a system of point-like masses due to their mutual gravitational interactions, including general relativistic effects. It uses a first-order post-Newtonian expansion and thus is valid in the limit where the velocities of the bodies are small compared to the speed of light and where the gravitational fields affecting them are correspondingly weak. Given a system of $\mathrm{N}$ bodies, labelled by indices $\mathrm{A}=1$, ..., $\mathrm{N}$, the barycentric acceleration vector of body $\mathrm{A}$ is given by: 


$$
\begin{aligned}
\vec{a}_{A}= & \sum_{B \neq A} \frac{G m_{B} \vec{n}_{B A}}{r_{A B}^{2}} \\
& +\frac{1}{c^{2}} \sum_{B \neq A} \frac{G m_{B} \vec{n}_{B A}}{r_{A B}^{2}}\left[v_{A}^{2}+2 v_{B}^{2}-4\left(\vec{v}_{A} \cdot \vec{v}_{B}\right)-\frac{3}{2}\left(\vec{n}_{A B} \cdot \vec{v}_{B}\right)^{2}\right. \\
& \left.-4 \sum_{C \neq A} \frac{G m_{C}}{r_{A C}}-\sum_{C \neq B} \frac{G m_{C}}{r_{B C}}+\frac{1}{2}\left(\left(\vec{x}_{B}-\vec{x}_{A}\right) \cdot \vec{a}_{B}\right)\right] \\
& +\frac{1}{c^{2}} \sum_{B \neq A} \frac{G m_{B}}{r_{A B}^{2}}\left[\vec{n}_{A B} \cdot\left(4 \vec{v}_{A}-3 \vec{v}_{B}\right)\right]\left(\vec{v}_{A}-\vec{v}_{B}\right) \\
& +\frac{7}{2 c^{2}} \sum_{B \neq A} \frac{G m_{B} \vec{a}_{B}}{r_{A B}}+O\left(c^{-4}\right)
\end{aligned}
$$

where:

$\vec{x}_{A}$ is the barycentric position vector of body $\mathrm{A}$

$\vec{v}_{A}=d \vec{x}_{A} / d t$ is the barycentric velocity vector of body $\mathrm{A}$

$\vec{a}_{A}=d^{2} \vec{x}_{A} / d t^{2}$ is the barycentric acceleration vector of body $\mathrm{A}$

$r_{A B}=\left|\vec{x}_{A}-\vec{x}_{B}\right|$ is the coordinate distance between bodies $\mathrm{A}$ and $\mathrm{B}$

$\vec{n}_{A B}=\left(\vec{x}_{A}-\vec{x}_{B}\right) / r_{A B}$ is the unit vector pointing from body $\mathrm{B}$ to body $\mathrm{A}$

$m_{A}$ is the mass of body $\mathrm{A}$.

$c$ is the speed of light

$G$ is the gravitational constant

and the big $\mathrm{O}$ notation is used to indicate that terms of order $\mathrm{c}^{-4}$ or beyond have been omitted.

Figure 16: Einstein-Infeld-Hoffmann equations

The coordinates used here are harmonic. The first term on the right hand side is the Newtonian gravitational acceleration at A; in the limit as $c \rightarrow \infty$, one recovers Newton's law of motion. It seems that this equation is perfect.

The Einstein-Infeld-Hoffmann equations are a weak field linearized version of GR that are appropriate when the curvature of spacetime is not too severe. This is certainly the case in the Solar System. The full nonlinear version of GR is only needed in situations in which the curvature of spacetime is severe, such as in the immediate vicinity of a black hole. The speed of gravitational effects in the Einstein-Infeld-Hoffmann equations is the speed of light, which is why $c$ appears in these equations. However, the corrections to planetary orbits, relative to Newtonian dynamics, predicted by the Einstein-InfeldHoffmann equations are very minor.

We can simplify this equation. We only consider the binary star system. There are only two objects $\mathrm{A}$ and $\mathrm{B}$. When the mass of $\mathrm{B}$ is much greater than the mass of $\mathrm{A}, v_{B}$ 
is approximately equal to 0 , so the equation is simplified to an equation with only A.

When the speed of the gravitational field is much greater than $c$, then it is Newtonian gravitation. $c \rightarrow \infty$, one recovers Newton's law of motion, there seems to be no problem.

When the speed of the gravitational field is much smaller than $c$, then the result of this equation has almost nothing to do with Newtonian gravity. $c \rightarrow 0$, force on $\mathrm{A}$ and $\mathrm{B}$ will both be very huge, which is very illogical!

The equations in question are the first few terms on an expansion, so obviously the equations do not give a sensible answer when $c$ goes to zero because the expansion breaks down. Therefore, I believe that the equation is a mathematical model established under the assumption that the gravitational speed is equal to $c$ to describe the planetary orbit under N-BODY. But it does not really describe the physical properties of gravity.

Einstein thought Gravitation is bending of space-time. everything moving with constant speed either it be light or anything else always follows geodesic. Then I want to ask a question: If the light is aimed at the center of the planet, what is the geodesic at this time? Will the light be bent? If our Earth also bends the surrounding space-time, then looking at the 2D space-time bending model, as shown in Figure 17(a), the light will never have a chance to reach the earth, and the light will only travel along the bent space-time, from the edge of the earth, go through. Obviously this $2 \mathrm{D}$ space-time bending model is incorrect, so will the 3D space-time bending model be correct? As shown in Figure 17(b), it can be seen that the surrounding space is recessed toward the center. The more severe the bending, the greater the impact of mass on space-time. It seems that the 3D model is closer to the truth. But if we express the magnitude of Newtonian gravitation at different positions in space as the distance from the source of gravity, then the final 3D model of Newtonian gravitation is almost the same as the 3D model of GR's space-time bending. It seems that Newton can also claim that universal gravitation bends space-time, but the fact is that it is only a mathematical model that uses distance to express the magnitude of gravitation. In this mathematical model, there are only coordinates of the direction and the magnitude of gravity, and no space coordinates $(\mathrm{x}, \mathrm{y}, \mathrm{z})$, space-time have never been bent. But Einstein obviously confuses these concepts. Not only does he use the degree of bending to express the magnitude of gravity, but also continues to retain the spatial coordinates $(\mathrm{x}, \mathrm{y}, \mathrm{z})$. GR is a wrong theory of gravity, which doomed the Einstein-Infield-Hoffman equation to be wrong. 


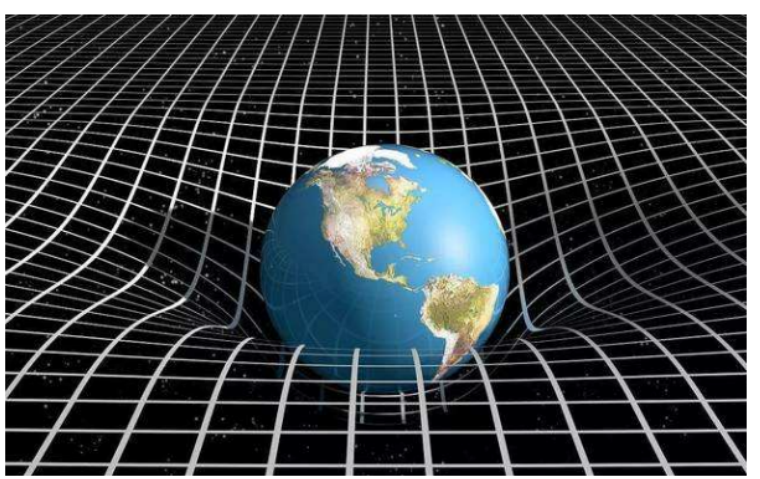

(a) 2D model

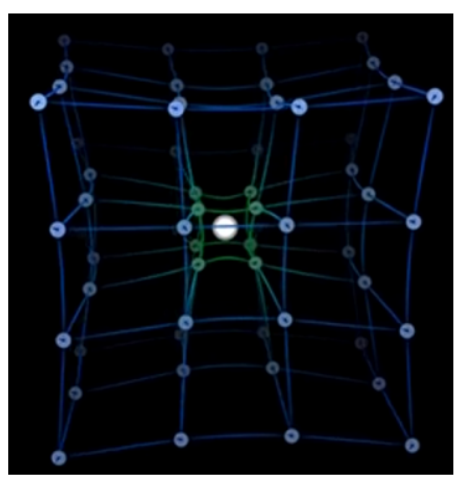

(b) 3D model

Figure 17: bending of space-time

\section{Conclusion}

The discovery of gravitational waves provides a new way for us to understand the universe, however, the speed of gravitational waves does not represent the speed of gravitational fields. The speed of action of gravitational fields is much greater than the speed of gravitational waves. As stated by Newton: Gravity is an action-at-a-distance force. Gravitational waves caused by the revolution of the Sun affect the orbits of planets and provide some planetary precession data. The general Doppler effect of gravitational waves also causes the planetary orbital mechanical energy to continue to increase slowly until the planet escapes from the solar system. Gravitational waves exist; the gravitational model under the influence of gravitational waves that we constructed was a physical model. Through the calculation of planetary orbital precession, the correctness of the gravity equation under the action of gravitational waves is verified, indicating that the gravitational physical model has research value. From Newton to Pierre-Simon Laplace have realized that the speed of gravity on objects is very huge. But this view is inconsistent with GR. From our analysis, GR is a wrong theory of gravity. The Newtonian model of universal gravitation under the action of gravitational waves is the correct way for humans to study the universe.

Finally, we also ask the following questions:

Is the acceleration of planetary orbits caused by the gravitational wave general Doppler effect related to the accelerated expansion of the universe?

Is there an association between the action-at-a-distance of the gravitational field and that in quantum mechanics? 


\section{References:}

[1] Fritz Rohrlich (25 August 1989). From Paradox to Reality: Our Basic Concepts of the Physical World. Cambridge University Press. pp. 28-. ISBN 978-0-521-37605-1.

[2] Newtonian Gravity http://farside.ph.utexas.edu/teaching/336k/Newtonhtml/node35.html

[3] "2018 CODATA Value: Newtonian constant of gravitation". The NIST Reference on Constants, Units, and Uncertainty. NIST. 20 May 2019. Retrieved 2019-05-20.

[4] "Doppler Shift". astro.ucla.edu.

[5] The Detection of Gravitational Waves using LIGO, B. Barish Archived 2016-03-03 at the Wayback Machine

[6] Abbott, R.; et al. (29 June 2021). "Observation of Gravitational Waves from Two Neutron Star-Black Hole Coalescences". The Astrophysical Journal Letters. 915 (1). doi:10.3847/2041-8213/ac082e. Retrieved 29 June 2021.

[7] Barry C. Barish, Caltech. The Detection of Gravitational Waves. Video from CERN Academic Training Lectures, 1996

[8] "Physics: Fundamental Forces and the Synthesis of Theory - Encyclopedia.com". www.encyclopedia.com.

[9] Einstein A., 1915, Erkl“"arung der Perihelbewegung des Merkur aus der allgemeinen Relativit"atstheorie. K" oniglich-Preußische Akad. Wiss. Berlin, pp. 831-839. English translation "Explanation of the perihelion motion of Mercury from general relativity theory" by R. A. Rydin with comments by A. A. Vankov, 1-34.

[10] Michal Krizek. Numerical experience with the finite speed of gravitational interaction. November 1999 Mathematics and Computers in Simulation 50(1-4):237-245 Follow journal, DOI: 10.1016/S0378-4754(99)00085-3

[11] The Speed of Gravity - What the Experiments Say December 1998Physics Letters A 250(s 1-3) DOI: 10.1016/S0375-9601(98)00650-1

[12] Einstein, A.; Infeld, L.; Hoffmann, B. (1938). "The Gravitational Equations and the Problem of Motion". Annals of Mathematics. Second series. 39 (1): 65-100. Bibcode:1938AnMat..39...65E. doi:10.2307/1968714. JSTOR 1968714. 